\title{
Radix Puerariae rebalances vasomotor factors and improves left ventricular diastolic dysfunction in patients with essential hypertension
}

\author{
YAN LIU $^{1 *}$, SU LIANG ${ }^{2 *}$, PEILI BU ${ }^{1}$, ERSHUN LIANG ${ }^{1}$, \\ FANGFANG YAN $^{3}$, YIFAN XING ${ }^{3}$ and PENGFEI ZHANG ${ }^{1}$
}

\begin{abstract}
${ }^{1}$ Department of Cardiology, Cardiovascular Remodeling and Function Research, Chinese Ministry of Education and Chinese Ministry of Health, and The State and Shandong Province Joint Key Laboratory of Translational Cardiovascular Medicine, Jinan, Shandong 250012; ${ }^{2}$ Department of Traditional Chinese Medicine, Jinan Maternity and Child Care Hospital, Jinan, Shandong 250001; ${ }^{3}$ Department of Traditional Chinese Medicine, Qilu Hospital of Shandong University, Jinan, Shandong 250012, P.R. China
\end{abstract}

Received August 05, 2019; Accepted January 30, 2020

DOI: $10.3892 /$ etm.2020.8746

\begin{abstract}
The aim of the present study was to validate the beneficial role of Radix Puerariae in rebalancing the plasma levels of endothelin, angiotensin II (AngII) and calcitonin gene-related peptide (CGRP) in patients with essential hypertension (EHT). A total of 150 patients with EHT were enrolled consecutively and randomized to receive antihypertensive drugs according to guideline-directed medical therapy (GDMT group) and GDMT plus Radix Puerariae (GDMT + RP group). The blood pressure was recorded biweekly. At baseline and at the end of the follow-up (12 weeks), the plasma levels of endothelin, AngII and CGRP were detected, whilst the left ventricular (LV) diastolic function was evaluated by echocardiography. At baseline, the two groups did not differ in terms of demographic characteristics and LV diastolic dysfunction. At the end of the follow-up, lower blood pressure was observed in the GDMT + RP compared with that in the GDMT group. The plasma levels of AngII and endothelin were also significantly lower in the GDMT + RP group. The plasma levels of CGRP increased significantly in the GDMT + RP compared with those in the GDMT group. The addition
\end{abstract}

Correspondence to: Professor Pengfei Zhang, Department of Cardiology, Cardiovascular Remodeling and Function Research, Chinese Ministry of Education and Chinese Ministry of Health, and The State and Shandong Province Joint Key Laboratory of Translational Cardiovascular Medicine, 107 Wenhuaxi Road, Jinan, Shandong 250012, P.R. China

E-mail: pengf-zhang@163.com

*Contributed equally

Key words: Radix Puerariae, left ventricular diastolic dysfunction, essential hypertension, endothelin, angiotensin II, calcitonin gene-related peptide, echocardiography of Radix Puerariae improved LV diastolic function, with the percentage of dysfunction decreasing to only $9 \%$, while this percentage remained significantly elevated (21\%) in the GDMT group. The results of the present study demonstrated that Radix Puerariae is able to regulate blood pressure and the plasma levels of endothelin, AngII and CGRP in patients with EHT. LV diastolic dysfunction was also improved, as detected by echocardiography.

\section{Introduction}

Essential hypertension (EHT) is one of the major risk factors for cardiovascular and cerebrovascular diseases. Approximately $62 \%$ of cerebrovascular diseases and $49 \%$ of ischemic heart diseases are attributed to suboptimal blood pressure worldwide. Pathologically, EHT may cause endothelial dysfunction and artery remodeling and accelerate atherosclerosis (1). Left ventricular (LV) diastolic dysfunction frequently presents as organ damage caused by EHT and this dysfunction may be overt in the early stages of EHT (2).

Endothelin (ET) is the strongest intrinsic vasoconstrictor and calcitonin gene-related peptide (CGRP) was proven to be the endogenous antagonist to ET. Angiotensin II (AngII) is a well-evidenced contributor to the pathophysiology of cardiovascular system disorders. These three proteins are widely distributed, particularly in the nervous and cardiovascular systems $(3,4)$. The balance among these factors regulates the proliferation and tone of vascular smooth muscle cells, as well as endothelial cell function. Increased plasma ET-1 and AngII levels, decreased CGRP levels and endothelial dysfunction were reported in spontaneously hypertensive rats (SHRs) and EHT patients $(5,6)$.

Antihypertensive drugs have been widely used in clinical practice, exerting protective effects on target organs beyond lowering the blood pressure. However, these effects are dose-dependent and the side effects are not negligible, and may include severe complications, e.g., renal failure or carotid stenosis. Traditional Chinese Medicine (TCM), an important 
part of complementary and alternative medicine, is widely accepted in Eastern Asia, particularly China, Japan and Korea. With TCM receiving an increasing amount of attention worldwide, studies are accumulating on the clinical efficacy of TCM formulations in the treatment of hypertension and the underlying mechanisms (7). Radix Puerariae, the dry root of the leguminous plant Puerariae (Willd.) Ohwi, the most effective active component of which is puerarin, is used as a TCM drug. It has been confirmed that Radix Puerariae is able to inhibit platelet aggregation and adhesion (8). It is also able to dilate peripheral arteries and improve the microcirculation. However, its effects on LV diastolic function and the balance among ET, AngII and CGRP have remained to be elucidated.

The present study reported on a prospective clinical trial investigating the utility of Radix Puerariae as an additive to guideline-directed medical treatment (GDMT) to alleviate LV diastolic dysfunction and improve the levels of plasma ET, AngII and CGRP in patients with EHT.

\section{Materials and methods}

Study design and population. The present study was a randomized controlled trial. It was performed according to the principles outlined in the Declaration of Helsinki and the General Principles for Clinical Research of New Drugs of Traditional Chinese Medicine issued by the State Drug Administration in 2015. The study protocol was approved by the Ethics Committee of Qilu Hospital of Shandong University (Jinan, China). Written informed consent was obtained from all participants.

The enrolled patients were aged 18-60 years and were initially diagnosed with EHT at the Qilu Hospital of Shandong University (Jinan, China) between November 2016 and June 2018. The exclusion criteria were as follows: Pregnancy, lactation, secondary hypertension, acute cerebral or cardiac events and severe renal or hepatic failure, contraindications to guideline-recommended antihypertensive drugs, tumors and psychiatric disorders.

Radix puerariae. The Radix Puerariae granules were purchased from China Resources Sanjiu Medical \& Pharmaceutical Co., Ltd. (batch no. 1611001w). The content of puerarin was $2.9 \%$ according to the outgoing test report for this batch, which meets the quality control criteria of the Chinese Pharmacopoeia Commission from 2015, which state that the puerarin content should not be $<2.4 \%$ (9).

Radix Puerariae is the dried root of Puerariae (Willd.) Ohwi. To date, 122 compounds have been isolated from Radix Puerariae, which are mainly classified into five categories: Flavonoids, puerosides, coumarins, triterpenoids and triterpene saponins. Among these, flavonoids, including puerarin, daidzin and daidzein, are the most abundant compounds.

Study procedure. Subjects were randomized into two groups, namely the GDMT group and the GDMT plus Radix Puerariae $(\mathrm{GDMT}+\mathrm{RP})$ group. GDMT included the monotherapy with any category among Ang-converting enzyme inhibitors, Ang receptor blockers, beta-receptor blockers, calcium channel blockers or diuretics which was prescribed to all patients, according to international guidelines on the management of hypertension (10). Measurement of blood pressure was performed biweekly during follow-up visits until the end of the 12th week. The dosage and combination of the abovementioned drugs were adjusted biweekly, if necessary, to achieve the blood pressure goal of $<140 / 90 \mathrm{mmHg}$. As for the strategy of drug adjustment, a second drug from the 5 categories mentioned above was added in sequence. If the blood pressure goal was not achieved with four drug categories, including diuretics, the dosage of the prescribed drugs was doubled or titrated. For patients in the GDMT + RP group, $15 \mathrm{~g}$ of Radix Puerariae granules was taken twice daily orally in addition to GDMT throughout the entire study. Echocardiography was performed and plasma levels of ET, AngII and CGRP were determined at baseline and the end of the follow-up. A flow chart of the study is provided in Fig. 1. The trial was terminated when the subject experienced a severe increase in blood pressure, developed severe comorbidities or exhibited poor compliance.

Blood pressure measurement. Blood pressure was measured using a mercurial sphygmomanometer. Blood pressure records were acquired for each patient from baseline to the end of the follow-up at biweekly intervals. At each visit, blood pressure was measured 3 times on the dominant arm in the sitting position after $10 \mathrm{~min}$ of rest and the mean reading was recorded.

Plasma ET, AngII and CGRP testing. The plasma levels of ET, CGRP and AngII were measured by radioimmunoassay at the Radiation and Immunology Center of Beijing People's Liberation Army General Hospital, (Beijing, China), according to methods described previously (11).

Detection of LV diastolic dysfunction. Echocardiography was performed using Vivid E90 equipment (GE Healthcare) with the M5s transducer (frequency, 1.5-4.5 MHz). The peak velocities of $\mathrm{E}$ and $\mathrm{A}$ waves across the mitral valve in diastole were acquired by pulsed-wave Doppler echocardiography. Mitral valve annulus velocity (e') was measured by tissue Doppler imaging. The mean value of the septal and lateral e' velocity was used to calculate the E/e' ratio (Fig. 2). The left atrial maximum volume indexed to body surface area $\left(\mathrm{LAv}, \mathrm{ml} / \mathrm{m}^{2}\right)$ was measured from apical four- and apical two-chamber views with the area-length method (Fig. 3). Body surface area (BSA) was calculated with the formula of Dubois and Dubois: BSA $\left(\mathrm{m}^{2}\right)=$ [weight $(\mathrm{Kg})^{0.425} \mathrm{x}$ height $(\mathrm{cm})^{0.725}$ x 0.007184 . Continuous-wave Doppler echocardiography was adopted to measure the systolic regurgitation velocity of the tricuspid valve (TRv) (Fig. 4). All parameters were measured 3 times and the mean value was calculated. E/e' $>14, \mathrm{LAv}>34 \mathrm{ml} / \mathrm{m}^{2}$ or TRv $>2.8 \mathrm{~m} / \mathrm{sec}$ were considered as indicators of abnormal LV diastolic function. LV diastolic dysfunction was diagnosed if at least two of the abovementioned indicators were positive, according to the guidelines of the American Society of Echocardiography (12).

Statistical analysis. Continuous variables are expressed as the mean \pm standard deviation and their normal distribution was tested with the one-sample Kolmogorov-Smirnov test. Categorical data are expressed as numbers and percentages. Differences between groups were analyzed by t-test for normally distributed continuous variables or Mann-Whitney U-test for non-normally distributed continuous variables. For 


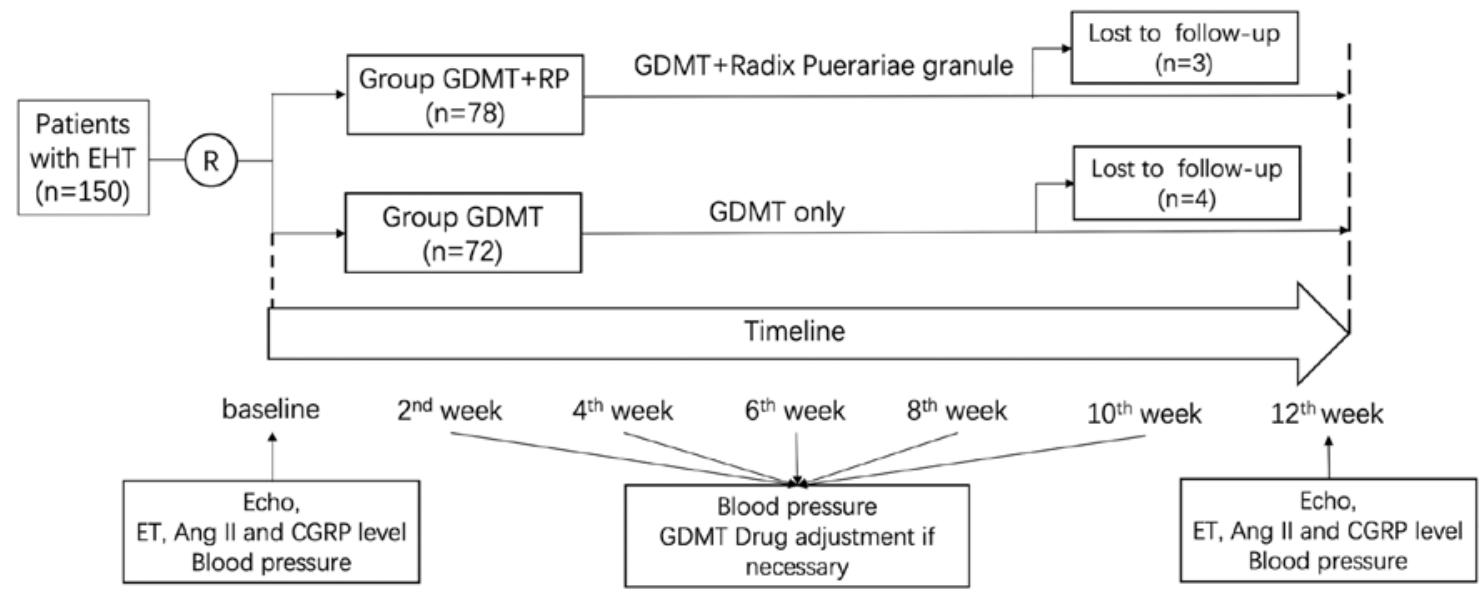

Figure 1. Flow chart of the study protocol. EHT, essential hypertension; R, randomization; RP, Radix Pueriae; GDMT, guideline-directed medical therapy; ET, endothelin; Ang, angiotensin; CGRP, calcitonin gene-related peptide.
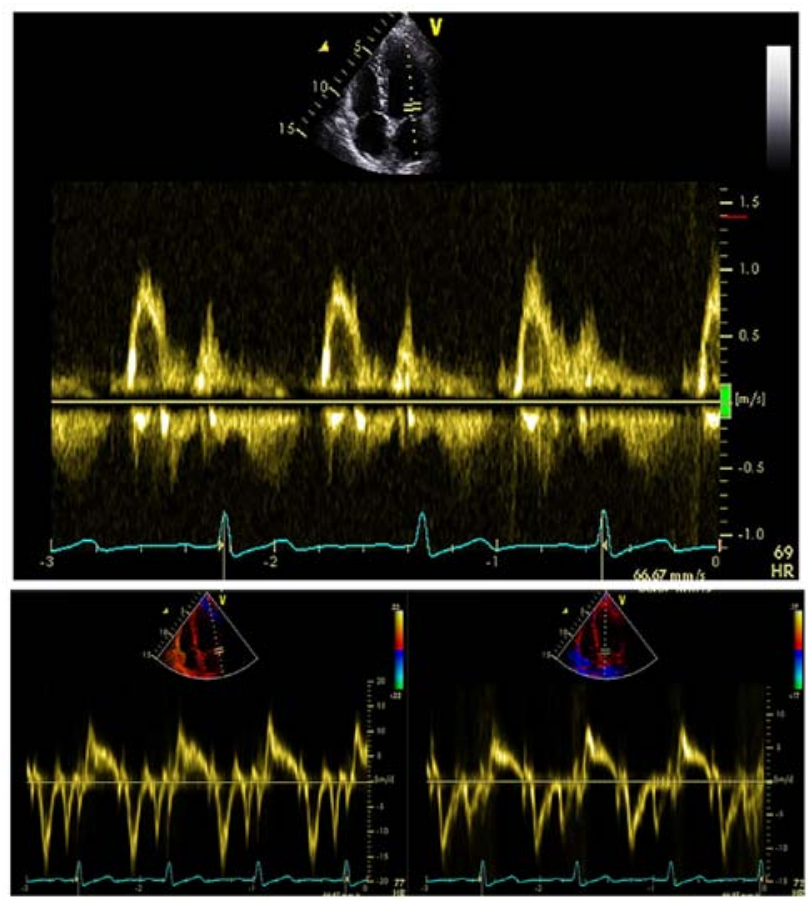

Figure 2. Calculation of E/e' by echocardiography. The E-value was acquired as the peak velocity of the $\mathrm{E}$ wave across the mitral valve (Image displayed in the upper part of the upper panel) in diastole by pulsed-wave Doppler echocardiography (upper panel). The value of e' was averaged with peak velocities of the e' wave acquired at the septal (lower left panel) and the lateral (lower right panel) mitral annulus by tissue Doppler echocardiography. HR, heart rate; s, seconds.

categorical data analysis, the $\chi^{2}$ test was performed. Analysis of covariance was used to analyze the interaction of Radix Puerariae and the blood pressure-lowering effect on LV diastolic function. All the analyses were performed with SPSS 13.0 (SPSS Inc.), and the $\mathrm{P}<0.05$ was considered to indicate a statistically significant difference.

\section{Results}

Demographic data. A total of 150 patients were included at the beginning of the study and 7 patients were excluded as they were lost to follow-up. Of the 143 patients included in the final analysis, 75 (52\%) were in the GDMT + RP group and 68 were in the GDMT group. There were no significant differences between the two groups in terms of patient sex, age, body mass index and smoking history. Blood pressure was also comparable between groups at baseline (Table I). LV diastolic dysfunction was detected in $32 \%(\mathrm{n}=24)$ of patients in the GDMT + RP group and in 29\% $(n=20)$ in the GDMT group.

Blood pressure. At the end of the follow-up, the blood pressure was lowered in the two groups compared with the baseline. In the two groups, a similar strategy with antihypertensive drugs was applied. Monotherapy with Ang receptor blockers and the combination of Ang receptor blockers with calcium channel blockers or diuretics were the most prevalent. Lower blood pressure $(124.3 \pm 8.4 / 88.4 \pm 4.2$ vs. $139.1 \pm 5.8 / 89.3 \pm 3.4 \mathrm{mmHg}$, $\mathrm{P}<0.001)$ was observed in the GDMT + RP group compared with that in the GDMT group. The systolic blood pressure-lowering effect was more prominent when Radix Puerariae was added (-23.6 vs. $-5.4 \mathrm{mmHg}$ with $\mathrm{P}<0.001$ for $\Delta$ systolic blood pressure, -5.6 vs. $-5.1 \mathrm{mmHg}$ for $\Delta$ diastolic blood pressure; Table II).

Plasma levels of ET, CGRP and AngII. The plasma levels of ET, CGRP and AngII did not significantly differ between the groups at baseline. At the end of the follow-up in the GDMT + RP group, the plasma levels of ET and AngII were found to be significantly reduced, whilst the plasma level of CGRP increased significantly compared with those at baseline. No such changes could be observed in the GDMT group. It was notable that at the end of the follow-up, the plasma levels of ET and AngII were also lower, whereas the level of CGRP was even higher in GDMT + RP group compared with those in the GDMT group (Fig. 5, Table III).

$L V$ diastolic dysfunction. A significant $\mathrm{E} / \mathrm{e}^{\prime}$ ratio reduction was only observed in the GMDT + RP group at the end of the study vs. baseline (10.75 \pm 1.86 vs. $9.55 \pm 0.84$, $\mathrm{P}<0.001$; Fig. 6 , Table IV). No significant difference was identified in left atrial volume at the end of week 12 in either group. The peak velocity of tricuspid regurgitation tended to be marginally decreased in 

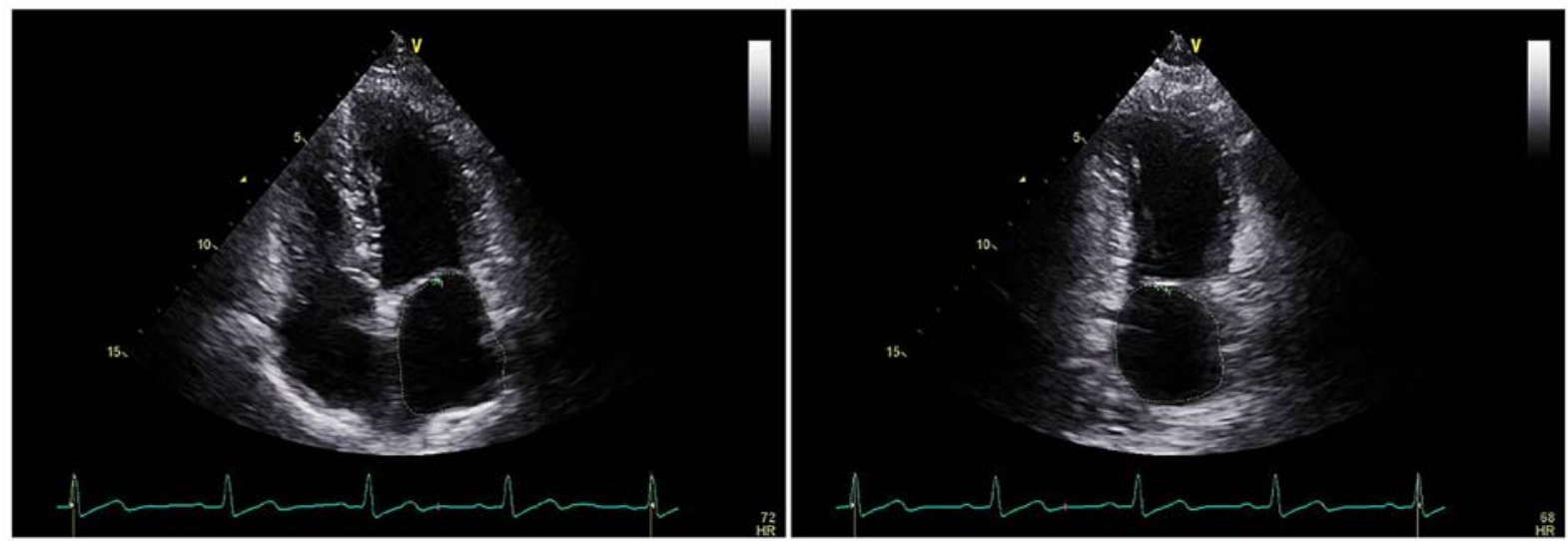

Figure 3. The left atrial maximum volume index was estimated using the area-length method. The volume (ml) was acquired from apical four- (right) and apical two-chamber (left) views in sequence. The two measurements were averaged and corrected for body surface area. HR, heart rate.

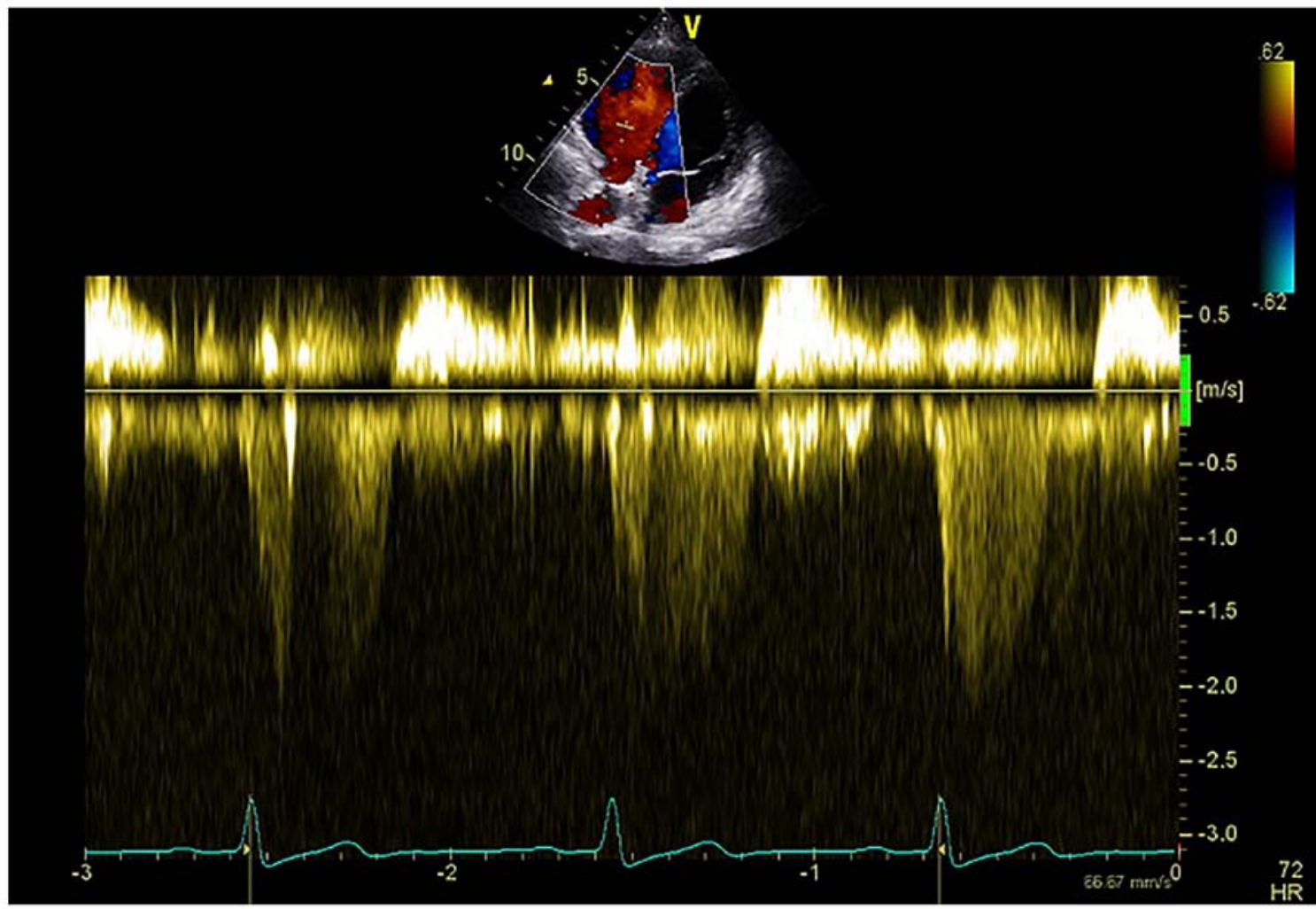

Figure 4. Systolic regurgitation velocity (m/sec) of the tricuspid valve (upper panel) was measured by continuous-wave Doppler. HR, heart rate; s, seconds.

the GDMT + RP group compared with that in the GMDT group (2.32 $\pm 0.51 v s$. 2.48 $\pm 0.49, \mathrm{P}=0.05$; Fig. 6, Table IV). However, the percentage of $\mathrm{LV}$ diastolic dysfunction was significantly reduced in the GDMT + RP group (32\% vs. 9\%, P=0.001). The improvement in LV diastolic dysfunction was not obvious in the GDMT group, as the percentage had only decreased from 29 to $21 \%$ at the end of the study $(\mathrm{P}=0.115)$. This beneficial effect of Radix Puerariae was independent of the blood pressure-lowering effect, as revealed by analysis of covariance.

\section{Discussion}

In addition to blood pressure control, management of hypertension should focus more on preventing damage of target organs, including the heart, brain and kidney. For the cardiovascular system, endothelial dysfunction and LV diastolic dysfunction are the preconditions of arterial and cardiac remodeling. ET, AngII and CGRP are important factors involved in the pathological process of cardiac dysfunction and remodeling. Antihypertensive drugs recommended by the guidelines on the management of adult hypertension have been indicated to exert a protective effect against cardiovascular remodeling (10). The present study demonstrated that Radix Puerariae, when used as an adjuvant to GDMT, had beneficial properties of improving LV diastolic function and rebalancing ET, AngII and CGRP levels. Although the blood pressure was lower in the GDMT + RP group, these beneficial effects of Radix Puerariae may be independent of its blood pressure-lowering action. 
Table I. Demographic data.

\begin{tabular}{|c|c|c|}
\hline Variable & Group GDMT + RP $(\mathrm{n}=75)$ & Group GDMT $(\mathrm{n}=68)$ \\
\hline Age (years) & $40.4 \pm 13.2$ & $39.8 \pm 11.5$ \\
\hline Male sex & $40(54)$ & $38(56)$ \\
\hline BMI $\left(\mathrm{kg} / \mathrm{m}^{2}\right)$ & $24.3 \pm 2.9$ & $24.8 \pm 2.9$ \\
\hline Systolic blood pressure (mmHg) & $147.9 \pm 13.3$ & $144.5 \pm 13.1$ \\
\hline Diastolic blood pressure (mmHg) & $94.0 \pm 9.2$ & $94.4 \pm 8.4$ \\
\hline Smoking history & $27(20)$ & $26(18)$ \\
\hline Left ventricular diastolic dysfunction & $24(32)$ & $20(29)$ \\
\hline \multicolumn{3}{|l|}{ Guideline-directed antihypertensive drugs } \\
\hline \multicolumn{3}{|l|}{ Monotherapy } \\
\hline ACEI & $5(7)$ & $4(6)$ \\
\hline ARB & $11(15)$ & $9(13)$ \\
\hline $\mathrm{CCB}$ & $7(9)$ & $5(7)$ \\
\hline $\mathrm{BB}$ & $3(4)$ & $2(3)$ \\
\hline Diuretics & $2(3)$ & (1) \\
\hline \multicolumn{3}{|l|}{ Double therapy } \\
\hline $\mathrm{ACEI}+\mathrm{CCB}$ & $8(11)$ & $9(13)$ \\
\hline ACEI + diuretics & $4(5)$ & $5(7)$ \\
\hline $\mathrm{ARB}+\mathrm{CCB}$ & $15(20)$ & $16(24)$ \\
\hline $\mathrm{ARB}+$ diuretics & $12(16)$ & $12(18)$ \\
\hline $\mathrm{CCB}+\mathrm{BB}$ & $3(4)$ & $2(3)$ \\
\hline \multicolumn{3}{|l|}{ Triple therapy } \\
\hline $\mathrm{ACEI}+\mathrm{CCB}+\mathrm{BB}$ & $2(3)$ & $1(1)$ \\
\hline $\mathrm{ARB}+\mathrm{CCB}+\mathrm{BB}$ & $3(4)$ & $2(3)$ \\
\hline
\end{tabular}

$\mathrm{P}>0.05$ for all the variables compared between groups. Values are expressed as $\mathrm{n}(\%)$ or the mean \pm standard deviation. BMI, body mass index; ACEI, angiotensin-converting enzyme inhibitor; ARB, angiotensin II receptor blocker; CCB, calcium channel blocker; BB, beta blockers; GDMT, guideline-directed medical therapy; RP, Radix Puerariae.

Table II. Differences in Blood pressure between the two groups at baseline and at the end of the follow-up.

\begin{tabular}{lccccrrr}
\hline & \multicolumn{3}{c}{ Group GDMT + RP $(\mathrm{n}=75)$} & & \multicolumn{3}{c}{ Group GDMT (n=68) } \\
\cline { 2 - 4 } \cline { 5 - 7 } Variable & Baseline & End & P-value & & Baseline & End & P-value \\
\hline SBP (mmHg) & $147.9 \pm 13.3$ & $124.3 \pm 8.4$ & $<0.001$ & & $144.5 \pm 13.1$ & $139.1 \pm 5.8^{\mathrm{a}}$ & $<0.001$ \\
DBP (mmHg) & $94.0 \pm 9.2$ & $88.4 \pm 4.2$ & $<0.001$ & & $94.4 \pm 8.4$ & $89.3 \pm 3.4$ & $<0.001$ \\
\hline
\end{tabular}

Values are expressed as the mean \pm standard deviation. SBP, systolic blood pressure; DBP, diastolic blood pressure; GDMT, guideline-directed medical therapy; RP, Radix Puerariae. ${ }^{\mathrm{a}} \mathrm{P}<0.001$ vs. Group GDMT+RP, end.

It was previously demonstrated that diastolic function may be reduced in the early stages of hypertension (13). This dysfunction may be accurately detected by echocardiography. Choi et al (14) observed a significant increase in left atrial volume index and $\mathrm{E} / \mathrm{e}^{\prime}$ ratio in a remodeled hypertension group. Furthermore, with the progression of myocardial remodeling, the gradual increase in the E/e' ratio became more overt, suggesting that diastolic function may be an important method for predicting LV remodeling in patients with hypertension. The tissue Doppler-derived e' velocity decreased with the decrease in LV relaxation. The present study demonstrated that, after treatment with Radix Puerariae in addition to standard antihypertensive therapy, the E/e' ratio decreased more significantly compared with that in the GDMT only group, suggesting reduced LV filling pressure. This result is in accordance with the results reported by Cai et al (15) and Gao et al (16). More importantly, it was evidenced that the LV diastolic dysfunction may be improved by combination of antihypertensive agents with Radix Puerariae.

ET is the most potent and longest-lasting vasoactive peptide secreted by vascular endothelial cells (17). ET binds 
Table III. Differences in plasma ET, CGRP and Ang II levels between groups at baseline and at the end of the follow-up.

\begin{tabular}{|c|c|c|c|c|c|c|}
\hline \multirow[b]{2}{*}{ Variable } & \multicolumn{3}{|c|}{ Group GDMT + RP $(n=75)$} & \multicolumn{3}{|c|}{ Group GDMT $(\mathrm{n}=68)$} \\
\hline & Baseline & End & P-value & Baseline & End & P-value \\
\hline ET & $74.43 \pm 5.84$ & $69.78 \pm 6.87$ & $<0.001$ & $75.7 \pm 5.61$ & $75.32 \pm 6.23^{\mathrm{a}}$ & 0.730 \\
\hline CGRP & $39.56 \pm 3.30$ & $44.69 \pm 2.65$ & $<0.001$ & $39.60 \pm 3.19$ & $39.31 \pm 3.08^{\mathrm{a}}$ & 0.565 \\
\hline AngII & $139.32 \pm 10.83$ & $125.08 \pm 9.47$ & $<0.001$ & $138.01 \pm 11.24$ & $134.78 \pm 8.78^{\mathrm{a}}$ & 0.064 \\
\hline
\end{tabular}

Values are expressed as the mean \pm standard deviation. ET, endothelin; CGRP, calcitonin gene-related peptide; AngII, angiotensin II; GDMT, guideline-directed medical therapy; RP, Radix Puerariae. ${ }^{\text {a }}<0.001$ vs. Group GDMT+RP, end.

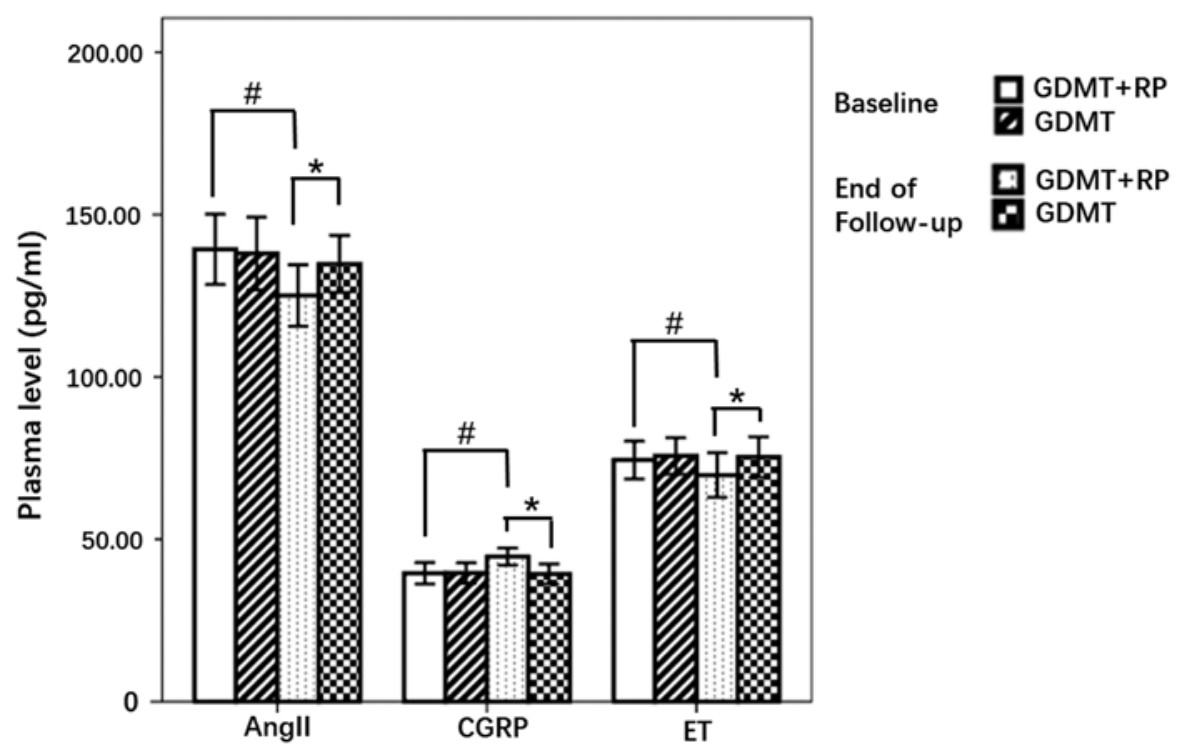

Figure 5. Plasma levels of ET, CGRP and AngII in each group at baseline and at the end of the follow-up. "P<0.05 compared with the GDMT + RP group at the end of follow-up. ${ }^{\text {P }}<0.05$ compared with the GDMT + RP group at baseline. ET, endothelin; CGRP, calcitonin gene-related peptide; AngII, angiotensin II; GDMT, guideline-directed medical therapy; RP, Radix Puerariae.

to its receptor ETA, thereby leading to impaired endothelial cell function and proliferation of vascular muscle cells and myocytes. It has been reported that there is a negative association between plasma ET levels and mitral valve E/A ratio, which is accompanied by LV diastolic dysfunction on pulsed-wave Doppler echocardiography. This supports the role of ET in LV diastolic function (18). Blockade of ET receptor may suppress myocardial hypertrophy and reduce the stiffness of the myocardium. Downregulation of ET expression may counteract the cardiac remodeling and prove beneficial in the therapy of heart failure by preserving the ejection fraction (19).

CGRP is currently known as the strongest endogenous vasodilating active polypeptide, which is widely distributed in the nervous system (20). It has been demonstrated that CGRP may antagonize the proliferation of vascular endothelial cells and smooth muscle cells induced by ET, thereby preventing the thickening of blood vessel walls and hypertension (21). Plasma ET levels were reported to be higher in patients with EHT compared with those in normal controls, whereas the CGRP level was reported to be lower (22). In the present study, it was observed that conventional antihypertensive drugs may decrease plasma AngII levels and increase the expression of CGRP, whilst
Radix Puerariae further reduced the ET levels. This suggests that conventional antihypertensive therapy combined with the application of Radix Puerariae may affect the plasma ET level in hypertensive patients, thereby improving endothelial cell function and exerting an antihypertensive therapeutic effect.

AngII is an important component of the renin-Ang system. When combined with type I receptors, it may cause pathological hypertrophy and fibrosis and generation of reactive oxygen species within the arterial wall and the myocardium. This contributes not only to increased blood pressure but also to cardiovascular remodeling $(23,24)$. As reported by Choi et al $(25)$, following stimulation by AngII in a mouse model, the LV diastolic function was hampered, as evidenced by increased $\mathrm{E} / \mathrm{e}^{\prime}$ ratio and decreased $\mathrm{e}^{\prime} / \mathrm{a}^{\prime}$ ratio determined by tissue Doppler echocardiography. AngII is also a promoter of ET expression and an inhibitor of CGRP expression (26). Radix Puerariae is able to reduce plasma AngII levels and downregulate the expression of AngII receptor type 1. Cai et al (27) reported that total flavone extracted from Radix Puerariae inhibited the plasma Ang-converting enzyme and renin activities in a dose-dependent manner, thus decreasing the blood pressure of $2 \mathrm{~K} 1 \mathrm{C}$ rats and SHRs. In the present study, AngII levels were significantly reduced in the Radix Puerariae group, 
Table IV. Differences in E/e', TRv and LAv between groups at baseline and at the end of follow-up.

\begin{tabular}{|c|c|c|c|c|c|c|}
\hline \multirow[b]{2}{*}{ Variables } & \multicolumn{3}{|c|}{ Group GDMT + RP $(n=75)$} & \multicolumn{3}{|c|}{ Group GDMT (n=68) } \\
\hline & Baseline & End & P-value & Baseline & End & P-value \\
\hline $\mathrm{E} / \mathrm{e}^{\prime}$ & $10.75 \pm 1.86$ & $9.55 \pm 0.84$ & $<0.001$ & $11.04 \pm 1.80$ & $11.00 \pm 1.79^{\mathrm{a}}$ & 0.900 \\
\hline $\operatorname{TRv}(\mathrm{m} / \mathrm{sec})$ & $2.36 \pm 0.51$ & $2.32 \pm 0.51$ & 0.641 & $2.40 \pm 0.50$ & $2.48 \pm 0.49^{\mathrm{b}}$ & 0.309 \\
\hline LAv (ml/BSA) & $29.41 \pm 5.47$ & $28.06 \pm 5.76$ & 0.143 & $29.06 \pm 4.91$ & $28.52 \pm 5.75$ & 0.554 \\
\hline Left ventricular dysfunction & $24(32)$ & $7(9)$ & 0.001 & $20(29)$ & $14(21)$ & 0.115 \\
\hline
\end{tabular}

Values are presented as mean \pm standard deviation or $\mathrm{n}(\%)$. TRv, tricuspid regurgitation velocity; LAv, left atrial maximum volume index; BSA, body surface area; GDMT, guideline-directed medical therapy; RP, Radix Puerariae. ${ }^{\mathrm{a}} \mathrm{P}<0.01$ and ${ }^{\mathrm{b}} \mathrm{P}=0.05$ vs. Group GDMT+RP, end.
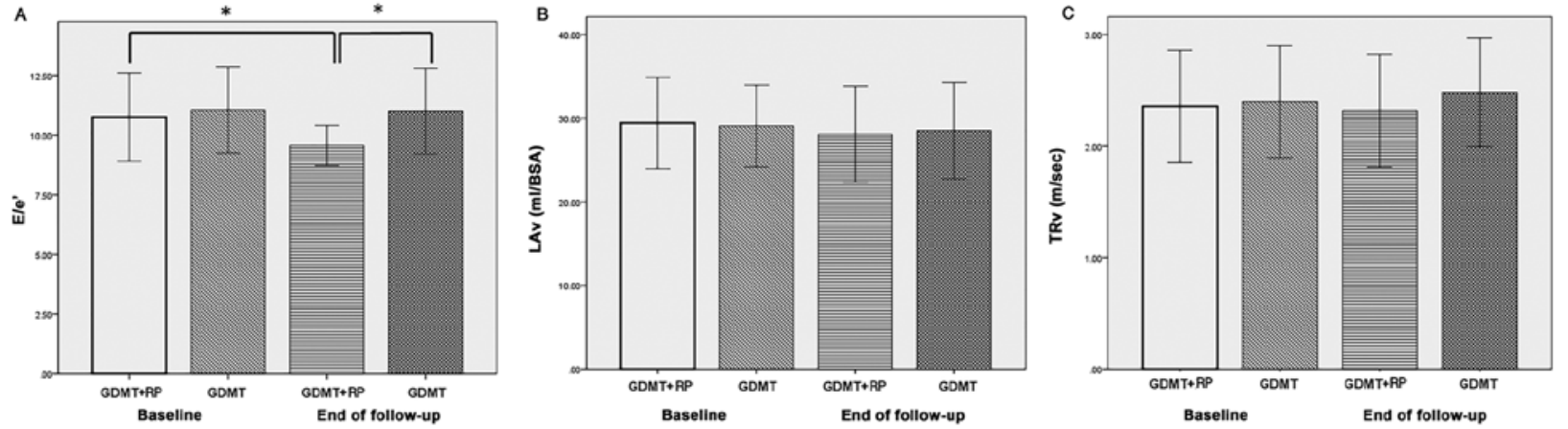

Figure 6. Changes of indices associated with left ventricular diastolic function. A: significant E/e' ratio reduction was observed with combination of Radix Puerariae. B: no significant difference was identified in terms of left atrial volume (LAv) at the end of follow-up in neither group. C: the peak velocity of tricuspid regurgitation (TRv) tended to be decreased in Group GDMT + RP compared with that in Group GMDT at the end of follow-up. GDMT, guidelinedirected medical therapy; RP, Radix Puerariae; BSA, body surface area. *P<0.001 vs. GDMT + RP group at the end of the follow-up.

\section{The mechanism of Radix Puerariae}

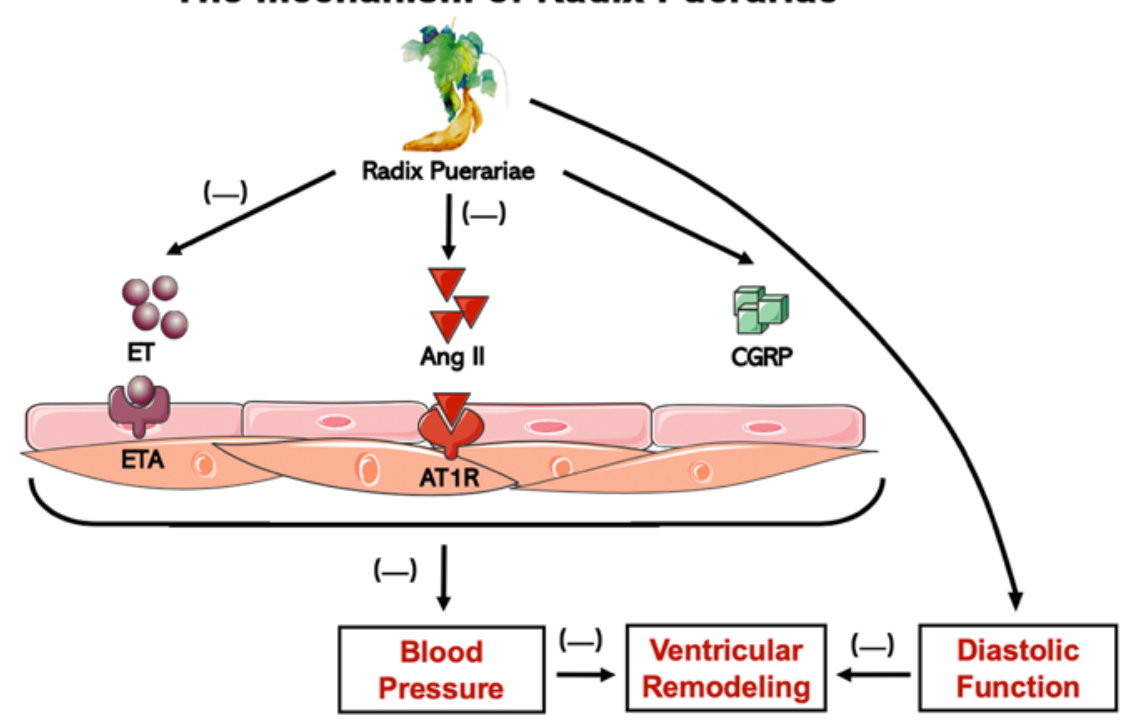

Figure 7. Mechanism of action of Radix Puerariae in patients with essential hypertension. ETA, endothelin receptor; CGRP, calcitonin gene-related peptide; AngII, angiotensin II; AT1R, angiotensin II receptor type 1.

which was also consistent with the results of Cai et al (15). It may be inferred that the renin-Ang system inhibitor drugs used in the present study are able to alleviate the effect of AngII. However, the percentage of patients receiving renin-Ang system inhibitors was comparable between the two groups of the present study. At the end of the follow-up, patients in the GDMT + RP group exhibited markedly lower levels of AngII, ET and blood pressure compared with those in GDMT group. The results suggest that Radix Puerariae was able to further reduce the levels of AngII in patients receiving conventional antihypertensive therapy. The reduction of AngII may act synergistically along with the reduction of ET caused by Radix Puerariae to regulate sympathetic 
nerve activity, achieving a more significant antihypertensive effect, and improve endothelial and LV diastolic dysfunction.

$\mathrm{Ng}$ et al (28) reported that an aqueous extract comprising Radix Puerariae exerted blood pressure-lowering effects in an in vivo SHR animal model and the mechanism may be explained by its endothelium-independent vasodilation via the opening of K(ATP), Kir and K(v) channels. These results all favor the conclusions of the present study. In addition, regulating potassium channels may also contribute to the antihypertensive effect of Radix Puerariae. The probable mechanism of action of Radix Puerariae in benefitting patients with EHT is provided in Fig. 7.

Although the biological mechanisms of TCM treatments remain largely elusive, various patients benefit from this practice. Recently, an increasing number of studies have been focusing on the standardization of TCM diagnosis and the use of gene sequencing technology for biomedical studies $(7,29)$. Combining TCM with precision medicine may broaden the field of medical research, help better understand disease mechanisms and promote advances in medicine.

In conclusion, the present study demonstrated that Radix Puerariae, as an adjunct to antihypertensive therapy, is able to regulate plasma endothelin, Ang II and CGRP levels in patients with EHT, significantly lower blood pressure and improve the clinical symptoms of hypertension. By improving LV diastolic function and preventing the occurrence of hypertensive ventricular remodeling, it may be a promising novel treatment for improving the clinical symptoms of hypertension and preventing the development of associated complications.

\section{Acknowledgements}

Not applicable.

\section{Funding}

The present study was supported by the National Science Foundation of China (grant no. 81571689), the Fundamental Research Funds of Shandong University (grant no. 2018JC009) and the National Key R\&D Program of China (grant no. 2017YFC1700502).

\section{Availability of data and materials}

The datasets generated and/or analyzed during the present study are available from the corresponding author on reasonable request.

\section{Authors' contributions}

PZ conceived and designed the study. YL and SL performed the experiments. ESL, YFX and FFY analyzed the data. PLB contributed to the echocardiographic examination. YL wrote the manuscript. All authors read and approved the final manuscript.

\section{Ethics approval and consent to participate}

The protocols of the present study were approved by the Ethics Committee of Qilu Hospital of Shandong University (Jinan,
China) and written informed consent was obtained from all participants.

\section{Patient consent for publication}

Not applicable.

\section{Competing interests}

The authors declare that they have no competing interests.

\section{References}

1. Bruno RM, Masi S, Taddei M, Taddei S and Virdis A: Essential hypertension and functional microvascular ageing. High Blood Press Cardiovasc Prev 25: 35-40, 2018.

2. Georgiopoulos G, Tsioufis CP, Kalos T, Magkas N, Rousos D, Chrysohoou C, Sarri G, Syrmali K, Georgakopoulos P and Tousoulis D: Serum uric acid is independently associated with diastolic dysfunction in apparently healthy subjects with essential hypertension. Curr Vasc Pharmacol 17: 99-106, 2019.

3. Mordi I, Mordi N, Delles C and Tzemos N: Endothelial dysfunction in human essential hypertension. J Hypertens 34: 1464-1472, 2016

4. Mihalj M, Tadzic R, Vcev A, Rucevic S and Drenjancevic I: Blood pressure reduction is associated with the changes in oxidative stress and endothelial activation in hypertension, regardless of antihypertensive therapy. Kidney Blood Press Res 41: 721-735, 2016.

5. Gao Y, Yang J and Wang S: Effects of telemetry implantation surgery on blood pressure and its underlying mechanism. Clin Exp Hypertens 38: 359-364, 2016.

6. Laffin LJ and Bakris GL: Endothelin antagonism and hypertension: An evolving target. Semin Nephrol 35: 168-175, 2015

7. Hao P, Jiang F, Cheng J, Ma L, Zhang Y and Zhao Y: Traditional Chinese medicine for cardiovascular disease: Evidence and potential mechanisms. J Am Coll Cardiol 69: 2952-2966, 2017.

8. Li H, Luo K and Hou J: Inhibitory effect of Puerariae Radix flavones on platelet-derived growth factor-BB-induced proliferation of vascular smooth muscle cells via PI3K and ERK pathways. Exp Ther Med 9: 257-261, 2015.

9. Chinese Pharmacopoeia Commission: Pharmacopoeia of the people's Republic of China (Part 1). China Med Sci Technol Press, pp333, 2015.

10. James PA, Oparil S, Carter BL, Cushman WC, DennisonHimmelfarb C, Handler J, Lackland DT, LeFevre ML, MacKenzie TD, Ogedegbe O, et al: 2014 evidence-based guideline for the management of high blood pressure in adults: Report from the panel members appointed to the eighth joint national committee (JNC 8). JAMA 311: 507-520, 2014.

11. Mao H, Gao F, Mao H, Zhang L, Guan W, Zhou Q, Zhang Y, Bai L and Zhan Y: Changes of plasma calcitonin gene-related peptide and other substances in healthy people who rush to high altitude. Zhong Guo Ying Yong Sheng Li Xue Za Zhi 22: 315-316, 2006 (In Chinese).

12. Nagueh SF, Smiseth OA, Appleton CP, Byrd BF III, Dokainish H, Edvardsen T, Flachskampf FA, Gillebert TC, Klein AL, Lancellotti P, et al: Recommendations for the evaluation of left ventricular diastolic function by echocardiography: An update from the American society of echocardiography and the European association of cardiovascular imaging. J Am Soc Echocardiogr 29: 277-314, 2016.

13. Marwick TH, Gillebert TC, Aurigemma G, Chirinos J, Derumeaux G, Galderisi M, Gottdiener J, Haluska B, Ofili E, Segers P, et al: Recommendations on the use of echocardiography in adult hypertension: A report from the European association of cardiovascular imaging (EACVI) and the American society of echocardiography (ASE). J Am Soc Echocardiogr 28: 727-754, 2015.

14. Choi J, Pyun WB and Shin GJ: The influence of the left ventricular geometry on the left atrial size and left ventricular filling pressure in hypertensive patients, as assessed by echocardiography. Korean Circ J 39: 145-150, 2009.

15. Cai RL, Li M, Xie SH, Song Y, Zou ZM, Zhu CY and Qi Y: Antihypertensive effect of total flavone extracts from Puerariae Radix. J Ethnopharmacol 133: 177-183, 2011. 
16. Gao Z, Wei B and Qian C: Puerarin injection for treatment of unstable angina pectoris: A meta-analysis and systematic review. Int J Clin Exp Med 8: 14577-14594, 2015.

17. Rautureau Y and Schiffrin EL: Endothelin in hypertension: An update. Curr Opin Nephrol Hypertens 21: 128-136, 2012.

18. Valero-Munoz M, Li S, Wilson RM, Boldbaatar B, Iglarz M and Sam F: Dual endothelin-A/endothelin-B receptor blockade and cardiac remodeling in heart failure with preserved ejection fraction. Circ Heart Fail 9: pii: e003381, 2016.

19. Kim YJ, Goh CW, Byun YS, Lee YH, Lee JB and Shin YO: Left ventricular hypertrophy, diastolic dysfunction, pulse pressure, and plasma ET-1 in marathon runners with exaggerated blood pressure response. Int Heart J 54: 82-87, 2013.

20. Kee Z, Kodji X and Brain SD: The role of calcitonin gene related peptide (CGRP) in neurogenic vasodilation and its cardioprotective effects. Front Physiol 9: 1249, 2018.

21. Watts SW: Serotonin and sensory nerves: Meeting in the cardiovascular system. Vascul Pharmacol 63: 1-3, 2014.

22. Inrig JK, Van Buren P, Kim C, Vongpatanasin W, Povsic TJ and Toto R: Probing the mechanisms of intradialytic hypertension: A pilot study targeting endothelial cell dysfunction. Clin J Am Soc Nephrol 7: 1300-1309, 2012.

23. Ramalingam L, Menikdiwela K, LeMieux M, Dufour JM, Kaur G, Kalupahana N and Moustaid-Moussa N: The renin angiotensin system, oxidative stress and mitochondrial function in obesity and insulin resistance. Biochim Biophy Acta Mol Basis Dis 1863: 1106-1114, 2017.

24. Wen KC, Lin SP, Yu CP and Chiang HM: Comparison of Puerariae Radix and its hydrolysate on stimulation of hyaluronic acid production in NHEK cells. Am J Chin Med 38: 143-155, 2010 .
25. Choi YS, de Mattos AB, Shao D, Li T, Nabben M, Kim M, Wang W, Tian R and Kolwicz SC Jr: Preservation of myocardial fatty acid oxidation prevents diastolic dysfunction in mice subjected to angiotensin II infusion. J Mol Cell Cardiol 100: 64-71, 2016.

26. Te Riet L, van Esch JH, Roks AJ, van den Meiracker AH and Danser AH: Hypertension: Renin-angiotensin-aldosterone system alterations. Circ Res 116: 960-975, 2015.

27. Cai SA, Hou N, Zhao GJ, Liu XW, He YY, Liu HL, Hua YQ, $\mathrm{Li} \mathrm{LR}$, Huang Y, Ou CW, et al: Nrf2 is a key regulator on puerarin preventing cardiac fibrosis and upregulating metabolic enzymes UGT1A1 in rats. Front Pharmacol 9: 540, 2018.

28. Ng CF, Koon CM, Cheung DW, Lam MY, Leung PC, Lau CB and Fung KP: The anti-hypertensive effect of Danshen (Salvia miltiorrhiza) and Gegen (Pueraria lobata) formula in rats and its underlying mechanisms of vasorelaxation. J Ethnopharmacol 137: 1366-1372, 2011.

29. Liu BC and Ji G: Sparking thinking: Studying modern precision medicine will accelerate the progression of traditional chinese medicine patterns. J Altern Complement Med 23: 502-504, 2017.

(i) (9) This work is licensed under a Creative Commons Attribution-NonCommercial-NoDerivatives 4.0 International (CC BY-NC-ND 4.0) License. 